\title{
Preparation, Characterization, and Crystallization of Naphthalene-Labeled Polypropylene
}

\author{
Jun YANG, ${ }^{\dagger}$ Guangxin Chen, Wanjun LIU, and Jingjiang LIU \\ State Key Laboratory of Poymer Physics and Chemistry, Changchun \\ Institute of Applied Chemistry, Chinese Academy of Sciences, \\ Changchun 130022, People's Republic of China
}

(Received April 10, 2000; Accepted August 18, 2000)

\begin{abstract}
Naphthalene-labeled polypropylene (PP) was prepared by melt reaction of maleic anhydride-graftedpolypropylene (PP-g-MA) with 1-aminonaphthalene in a Barabender mixer chamber. The structure of the product was analyzed with fourier transform infrared (FT-IR), ultraviolet (UV) and fluorescence. The results showed that naphthyl groups grafted onto the PP molecular chains through the imide bonds formed between MA and 1-aminonaphthalene. The content of the chromophores was $1.8 \times 10^{-4} \mathrm{~mol} \mathrm{~g}^{-1}$ measured by elemental analysis. Isothermal crystallization behavior was studied by differential scanning calorimeter (DSC). Labeled PP had a higher crystallization rate than PP-gMA. Wide-angle X-Ray diffraction (WAXD) analysis revealed that labeled PP had higher crystallinity than PP-g-MA. KEY WORDS Melt Reaction / Excimer Fluorescence / Polypropylene / Naphthalene /
\end{abstract}

Excimer formation has been widely used to study polymer physics and processing. This fluorescence quenching can give information about miscibility of polymer blends, ${ }^{1}$ residual strains in polymer materials, ${ }^{2}$ chain folding, ${ }^{3}$ distribution of functional groups grafted onto a polymer backbone, ${ }^{4}$ chain interactions, ${ }^{5}$ and conformation and dynamics of polymers in dilute solution. ${ }^{6}$ These experiments all require polymers to which appropriate fluorescent groups are attached. Pyrene and naphthalene are commonly fluorescent probes for this purpose because of relatively higher quantum yield.

Many routes have been reported for the preparation of polymer probes containing small amounts of fluorescent chromophores. Free-radical copolymerization and polymer functional reactions in solution are the main methods. Cheng ${ }^{7}$ prepared copolymers of 1-naphthylmethyl methacrylate and methyl methacrylate by free radical polymerization. $\mathrm{Li}^{5}$ prepared pyrenyl-labeled polystyrene by copolymerization of styrene and a py-monomer. The py-monomer is synthesized from 1-pyrenylmethanol with $\mathrm{m} / \mathrm{p}$-chloromethyl styrene. Mathew et al. ${ }^{3}$ synthesized the pyrenyl-labeled polystyrene (PS) with the same structure. However, they followed the second route. PS was chloromethylated first and then 1-pyrenemethanol was added. Similarly, Liaw, et al. ${ }^{8}$ incorporated naphthyl group into a cationic copolymer and its photophysical response was used to probe solution behavior on the microscopic level. Zhao ${ }^{1}$ prepared naphthalene-labeled linear low density polyethylene (LLDPE) by grafting maleic anhydride (MA) onto LLDPE first and then the product reacted with aminonaphthalene in xylene solution. Jao ${ }^{9}$ reacted maleated ethylene-propylene copolymer with 1pyrenebutylhydrazine in mineral lubricating oil solution to introduce the fluorescent probes.

This article reports the preparation of naphthalenelabeled polypropylene (PP) by melt reaction of maleated $\mathrm{PP}$ with 1-aminonaphthalene. Characterization indicated the succinic anhydride groups on the PP-g-MA chains to react with 1-aminonaphthalene thoroughly.
DSC analysis of isothermal crystallization revealed that labeled PP has a higher crystallization rate than PP-gMA as well as a higher crystallinity measured by WAXD. To our knowledge, labeling fluorescent chromophores to a polymer in melt instead of in solution is scarcely reported. The advantage of melt reaction is that this method can produce a wide variety of products than the reaction in solution.

\section{EXPERIMENTAL}

Isotactic polypropylene (model 1300) with melt index of $1.20 \mathrm{~g} / 10 \mathrm{~min}$ was purchased from Yanshan Petrolchemicals Co. (Beijing China). Dicumyl peroxide (DCP) and maleic anhydride (MAH) were of reagent grade and used without further purification. 1-Aminonaphthalene was purchased from Xingzhong Chemical Factory in Shanghai, China.

PP-g-MA was first manufactured by melt mixing of PP melt and MAH in the presence of DCP at $180^{\circ} \mathrm{C}$ in a Barabender mixing chamber as described in the literature. ${ }^{10}$ The content of maleic anhydride in PP-g-MA was $1.7 \mathrm{wt} \%$ as measured by titration. Naphthalene-labeled PP was prepared by melt mixing of PP-g-MA with 1aminonaphthalene in excess in a Barabender mixing chamber. Mixing temperature was controlled at $180^{\circ} \mathrm{C}$ and the rotation speed was $50 \mathrm{rpm}$. Identical processing was performed for PP-g-MA sample without addition of 1-aminonaphthalene, to obtain a comparative sample with the same thermal history. Labeled PP and unlabeled PP-g-MA were purified by repeated dissolving and precipitation using a system of xylene and methanol, solvent and precipitation agent, respectively, under a nitrogen atmosphere.

Naphthalene-labeled PP was analyzed on a JFS 66 V FT-IR spectrophotometer. UV absorption spectra were obtained with an UV-2101PC spectrophotometer and film samples of $30 \mu \mathrm{m}$ in thickness were used. Steadystate fluorescence spectra were measured using a SPEX

\footnotetext{
${ }^{\dagger}$ To whom correspondence should be addressed (E-mail: nkuyj@263.net).
} 


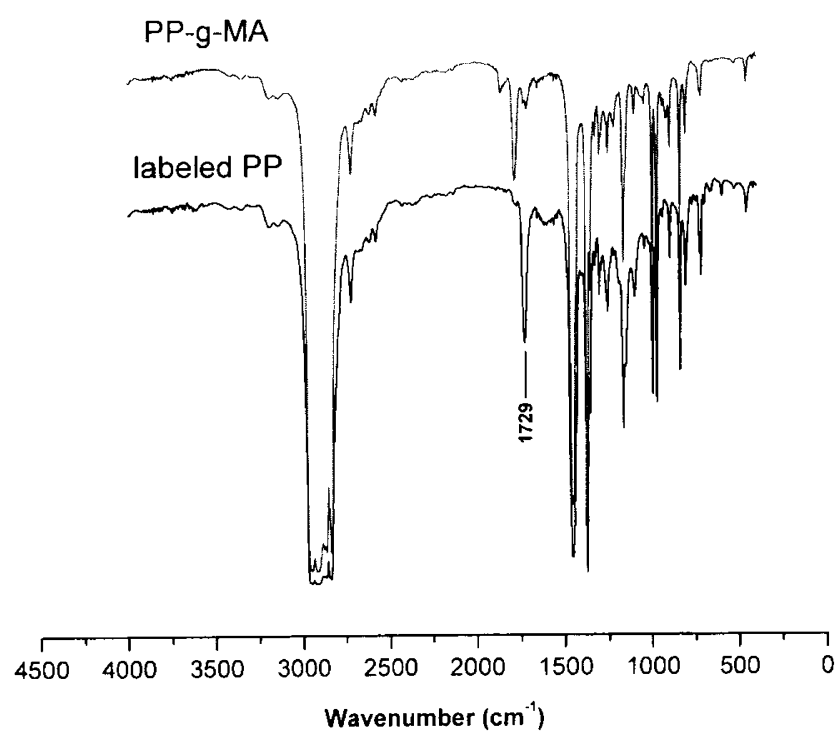

Figure 1. FT-IR spectra of PP-g-MA and labeled PP.

212 spectrometer. Measurement was taken with frontface geometry from polymer films at an excitation wavelength of $280 \mathrm{~nm}$. Elemental analysis was performed on a Vario EL elemental analyzer made in Germany.

Crystallization kinetic experiments were carried out using a Perkin-Elmer DSC-7 calibrated with indium and zinc standards. Isothermal crystallization kinetics were performed by first heating the sample quickly (at $80^{\circ} \mathrm{C}$ $\min ^{-1}$ ) to $220^{\circ} \mathrm{C}$ in the DSC, holding it at $220^{\circ} \mathrm{C}$ for 10 min to eliminate residual and small nuclei that might act as seed crystals, and then cooling (at $-80^{\circ} \mathrm{C}$ min $^{-1}$ ) the melt to the designated crystallization temperatures, $T_{c}$. Exothermal curves of heat flow as a function of time were recorded and investigated. All operations were performed under a nitrogen purge. Sample weights were from $4-6 \mathrm{mg}$. Relative crystallinity $X_{\mathrm{t}}$ was determined by

$$
X_{\mathrm{t}}=\frac{\int_{t_{0}}^{t}\left(\frac{\mathrm{d} H}{\mathrm{~d} t}\right) \mathrm{d} t}{\int_{t_{0}}^{t_{\infty}}\left(\frac{\mathrm{d} H}{\mathrm{~d} t}\right) \mathrm{d} t}
$$

where $\mathrm{d} H / \mathrm{d} t$ is the rate of heat flow, $t_{0}$ is the time at which the sample attained the isothermal condition, as indicated by a flat base line on the thermal curve after the initial spike.

Wide-angle X-Ray diffraction profiles of labeled PP and PP-g-MA were obtained using a PW 1700 Philips diffractometer with $\mathrm{Cu}-K_{\alpha}$, Ni-filtered radiation. The measurement condition was $40 \mathrm{KV}$ and $30 \mathrm{~mA}$.

\section{RESULTS AND DISCUSSION}

\section{Structure Analysis}

FT-IR spectra of PP-g-MA and labeled PP are shown in Figure 1. Absorbance bands at $1863 \mathrm{~cm}^{-1}$ and 1785 $\mathrm{cm}^{-1}$ for the carbonyl group of the succinic anhydride attached to the PP backbone were observed in the spectrum of PP-g-MA. These two bands disappeared in the spectrum of labeled PP and a new absorbance band at

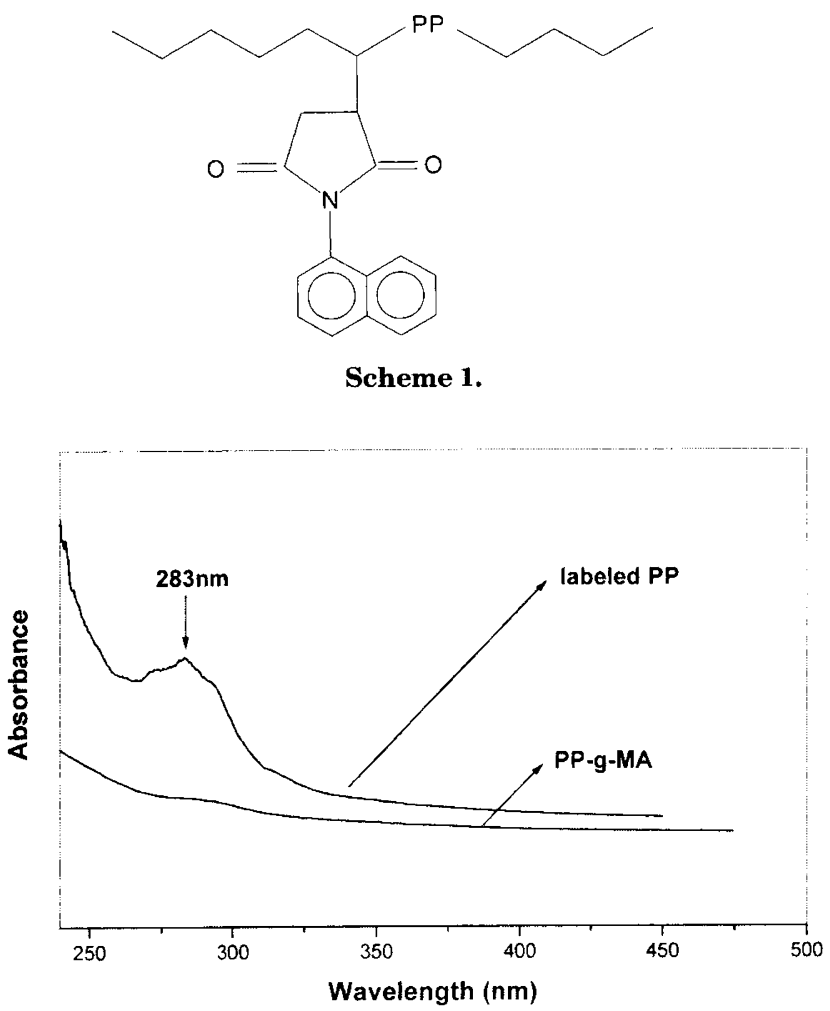

Figure 2. UV absorption spectra of PP-g-MA and labeled PP.

$1729 \mathrm{~cm}^{-1}$ is observed. This new band is attributed to the absorption of the imide group formed between the pendant succinic anhydride and 1-aminonaphthalene, as shown in Scheme 1. Similar results were obtained by Jao, et $a l .{ }^{9}$ In their report, the new band was observed at $1730 \mathrm{~cm}^{-1}$, as a proof of the quantitative conversion of the succinic anhydride to imide. In our work, the clear band at $1729 \mathrm{~cm}^{-1}$ and disappearance of the two bands at $1863 \mathrm{~cm}^{-1}$ and $1785 \mathrm{~cm}^{-1}$ give evidence that the attached succinic anhydride quantitatively reacts with 1aminonaphthalene.

UV absorption spectra of PP-g-MA and labeled PP are shown in Figure 2. The absorption between 270-300 $\mathrm{nm}$ is the typical absorption of naphthalene monomers. The peak position is at $283 \mathrm{~nm}$. In the spectrum of PP-gMA, characteristic absorption bands of naphthalene are not observed in the wavelengths measured in this work. The presence of naphthyl groups in the labeled PP was confirmed from these results.

Figure 3 gives uncorrected emission spectra of PP-gMA and labeled PP at excitation wavelength of $280 \mathrm{~nm}$. In the spectrum of labeled PP, the peak at $337 \mathrm{~nm}$ corresponds to the isolated naphthalene monomer and broad band at $400 \mathrm{~nm}$ corresponds to the excimer complex. Excimer emission is often structureless relative to the fine structure of monomer emission. ${ }^{11}$ In the spectrum of PPg-MA, the peaks mentioned above were not observed. This indicates again that the labeled PP contains naphthalene chromophores.

The chromophore content of labeled polymer is usually determined by UV absorption in solution. In this work, unfortunately, no solvent for labeled PP was found at ambient temperature. Hence, an elemental analysis was used to determine the content of naphthyl groups at- 


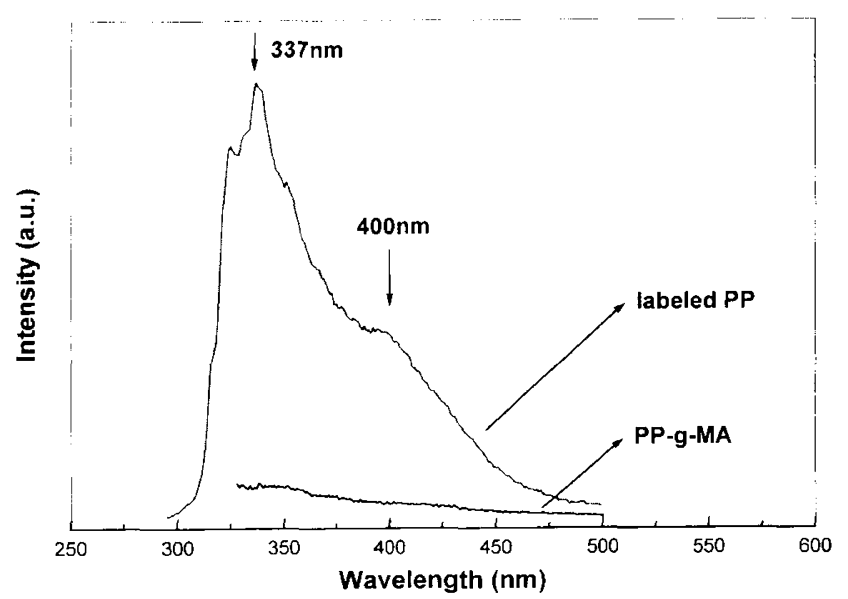

Figure 3. Typical fluorescence spectra of PP-g-MA and labeled PP.

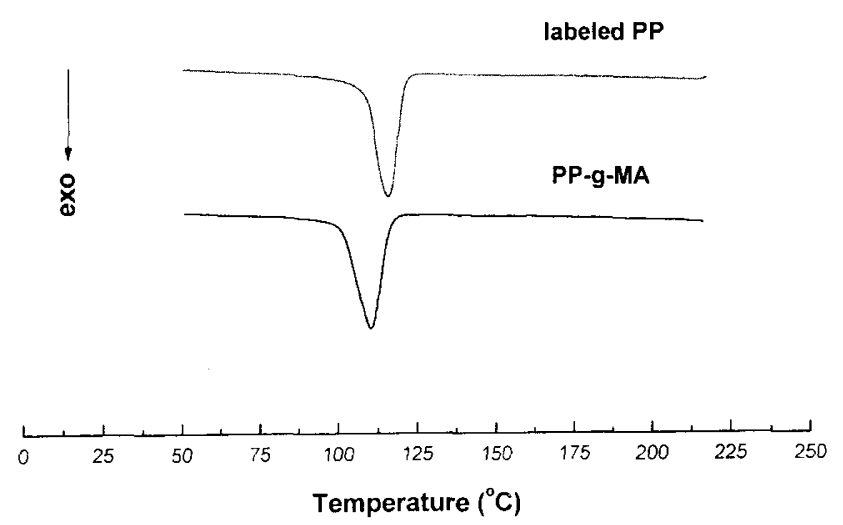

Figure 4. Crystallization exotherms on cooling at $10 \mathrm{~K} \mathrm{~min}^{-1}$ for PP-g-MA and labeled PP.

tached to PP chains. Oxygen and nitrogen were measured repeatedly and good reproducibility was obtained. $\mathrm{O}$ and $\mathrm{N}$ in the labeled $\mathrm{PP}$ were $0.57 \mathrm{wt} \%$ and $0.28 \mathrm{wt} \%$ on the average, i.e., $3.6 \times 10^{-4} \mathrm{~mol} \mathrm{~g}^{-1}$ and $1.8 \times 10^{-4}$ $\mathrm{mol} \mathrm{g}^{-1}$, respectively. The molar ratio of $\mathrm{O} / \mathrm{N}$ in the labeled PP is 2 to 1 . This means that all succinic anhydride units react with 1-aminonaphthalene (Scheme 1). The content of naphthyl groups attached to the PP backbone is equal to that of element $\mathrm{N}$ in labeled PP, $1.8 \times$ $10^{-4} \mathrm{~mol} \mathrm{~g}^{-1}$. This value is in agreement with that of MA in PP-g-MA as measured by titration method.

\section{Crystallization Behaviors}

Figure 4 gives crystallization exotherms for PP-g-MA and labeled PP. Curves of thermal analysis were recorded by heating the purified sample from room temperature to $220^{\circ} \mathrm{C}$ rapidly and holding it there for $10 \mathrm{~min}$ to remove all residues of crystallinity, and then cooling it down to room temperature at $10^{\circ} \mathrm{C} \mathrm{min}-1$. The crystallization peak for PP-g-MA is about $110^{\circ} \mathrm{C}$, while the peak for labeled $\mathrm{PP}$ is shown at about $116^{\circ} \mathrm{C}$. Reduction of the supercooling degree indicates that the pendant naphthyl group induces the crystallization of PP at higher temperatures.

The Avrami equation was used to analyze the isothermal crystallization of labeled PP as shown in Figure 5. PP-g-MA exhibited similar trends and the curves are omitted. Crystallization was retarded by raising the

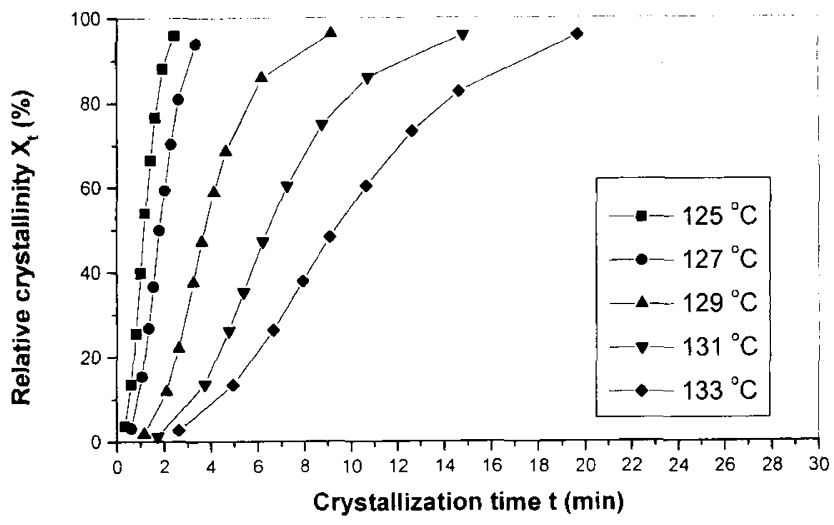

Figure 5. Development of relative crystallinity $X_{\mathrm{t}}$ with crystallization time for isothermal melt crystallization of labeled PP.

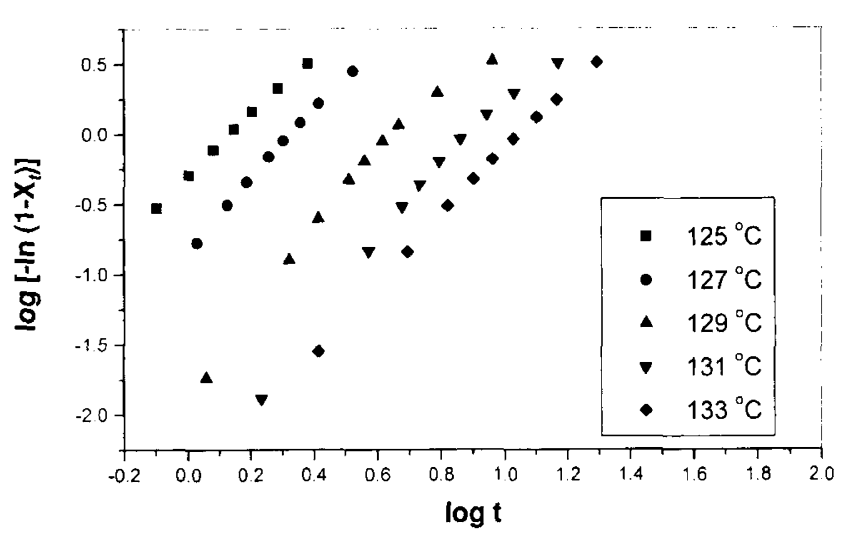

Figure 6. Plots of $\log \left[-\ln \left(1-X_{\mathrm{t}}\right)\right] v s$. $\log t$ (in min) for isothermal melt crystallization of labeled PP at different temperatures.

Table I. Kinetic parameters for isothermal melt crystallization of PP-g-MA and labeled PP

\begin{tabular}{ccccc}
\hline Sample & $T_{\mathrm{c}} /{ }^{\circ} \mathrm{C}$ & $n$ & $K$ & $t_{1 / 2} / \mathrm{min}$ \\
\cline { 2 - 5 } PP-g-MA & 121 & 2.6 & $5.6 \mathrm{e}-2$ & 2.6 \\
& 123 & 2.6 & $1.8 \mathrm{e}-2$ & 4.0 \\
& 125 & 2.5 & $1.8 \mathrm{e}-2$ & 4.2 \\
& 127 & 2.6 & $2.5 \mathrm{e}-3$ & 8.7 \\
& 129 & 2.4 & $6.8 \mathrm{e}-4$ & 18.4 \\
\hline \multirow{3}{*}{ Labeled $i$ PP } & 125 & 2.4 & $5.0 \mathrm{e}-1$ & 1.1 \\
& 127 & 2.8 & $1.4 \mathrm{e}-1$ & 1.8 \\
& 129 & 3.1 & $1.2 \mathrm{e}-2$ & 3.7 \\
& 131 & 3.0 & $2.6 \mathrm{e}-3$ & 6.3 \\
\hline
\end{tabular}

crystallization temperature $\left(T_{\mathrm{c}}\right)$. The well-known double logarithmic plots of $\log \left[-\ln \left(1-X_{\mathrm{t}}\right)\right]$ vs. $\log t$ are shown in Figure 6. Each curve showed an initial linear portion (the primary crystallization stage), subsequently tending to level off. This deviation was considered due to the secondary crystallization. Avrami parameters $n$ and $K$ for isothermal melt crystallization, determined from the linear portions in Figure 6 are listed in Table I. The crystallization half life, defined as the time at which the extent of crystallization is $50 \%$ complete, is determined from the measured kinetic parameters, i.e.,

$$
t_{\frac{1}{2}}=\left(\frac{\ln 2}{K}\right)^{\frac{1}{n}}
$$




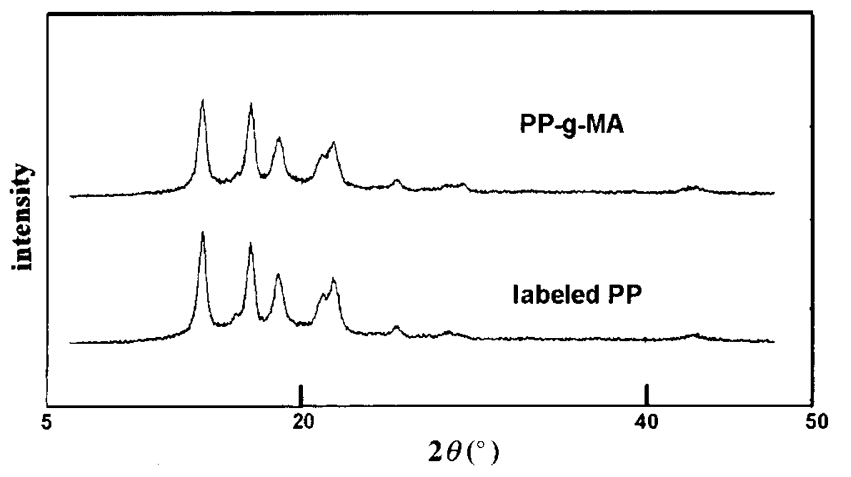

Figure 7. WAXD patterns of PP-g-MA and labeled PP.

Usually, the crystallization rate constant $K$ is determined as the reciprocal of $t_{1 / 2}$, i.e., $K=\left(t_{1 / 2}\right)^{-1}$. Values are presented in Table I. As seen from Table I, labeled PP has higher crystallization rate constants than PP-g-MA at the same crystallization temperatures. This means that the attached naphthyl groups on the PP backbone restrict the motion of $\mathrm{PP}$ segments and facilitate nuclei formation at higher temperatures. An alternative interpretation is that the naphthyl group aggregates play a part of heterogeneous nuclei for the crystallization. Aggregation of naphthyl groups facilitates the excimer formation, showing an evident excimer emission in Figure 3 .

Figure 7 gives WAXD patterns of labeled PP and PP-gMA. Typical $\alpha$-form crystal of $i$ PP was observed in each sample. The degree of crystallinity of the $\alpha$-form of PP can be obtained by eq $3:^{12}$

$$
W_{\mathrm{c}, \mathrm{x}}=\frac{I_{\mathrm{c}}}{I_{\mathrm{c}}+1.25 I_{a}} \times 100 \%
$$

where $W_{c, x}$ is the degree of crystallinity of $\alpha$-PP, $I_{\mathrm{a}}$ integral intensity of the amorphous peak, $I_{\mathrm{c}}=I_{110}+1.63 I_{040}$ $+2.14 I_{130}+3.51 I_{041}$, and $I_{110}, I_{040}, I_{130}$, and $I_{041}$, integral intensities of corresponding crystalline peaks. The crystallinities for labeled PP and PP-g-MA measured by WAXD are $52.1 \%$ and $44.6 \%$, respectively. This indicates that incorporation of naphthyl groups not only increases the crystallization rate but also the crystallinity of $\alpha$ PP.

This work is in progress at our lab.

\section{CONCLUSION}

By melt reaction, chromophore naphthalene was introduced into PP chains through the imide bonds formation between MA in PP-g-MA and 1-aminonaphthalene. The structure of the labeled PP was analyzed by means of FT-IR, UV, and fluorescence technique. Naphthyl groups were grafted onto the PP backbones. Naphthyl groups was determined as $1.8 \times 10^{-4} \mathrm{~mol} \mathrm{~g}^{-1}$ by elemental analysis. DSC analysis revealed that labeled PP has higher rate of crystallization than PP-g-MA at the same crystallization temperatures. The incorporation of pendent naphthyl groups thus facilitates crystallization at higher temperatures. WAXD results showed that labeled PP has higher crystallinity than PP-g-MA.

Acknowledgment. This work is a part of the fundamental research of general polymer materials with high performance, No. G 1999064800 , subsidized by the special funds for major basic research project.

\section{REFERENCES}

1. H. Zhao, Z. Lei, and B. Huang, Polym. J., 30, 149 (1998).

2. T. Ikawa, T. Shiga, and A. Okada, J. Appl. Polym. Sci., 66, 1569 (1997).

3. A. Mathew, H. Siu, and J. Duhamel, Macromolecules, 32, 7100 (1999).

4. S. Nemeth, T. Jao, and J. Fendler, J. Polym. Sci., Part B: Polym. Phys., 34, 1723 (1996).

5. X. Li, G. Wang, and B. He, Polym. Int., 48, 529 (1999).

6. J. Duhamel, A. Yekta, M. A. Winnik, T. C. Jao, M. K. Mishra, and I. D. Rubin, J. Phys. Chem., 97, 13708 (1993).

7. L. Cheng, G. Wang, and M. A. Winnik, Polymer, 31, 1611 (1990).

8. D. Liaw, C. Huang, and E. Kang, J. Polym Sci., Part B: Polym. Phys., 36, 1 (1998).

9. T. Jao, M. Mishra, J. Duhamel, and M. A. Winnik, J. Polym. Sci., Part B: Polym. Phys., 33, 1173 (1995).

10. R. M. Ho, A. C. Su, C. H. Wu, and S. I. Chen, Polymer, 34, 3264 (1993).

11. G. Wang and L. Chen, Huaxue Tongbao, 1, 1 (1989).

12. Z. Mo, in "Application of Modern Analytical Technique to Polymers", R. Wu, Ed., Shanghai Science and Technology Press, Shanghai, 1987, p 300. 